\title{
Biocompatibility of biological material polylactic acid with stem cells from human exfoliated deciduous teeth
}

\author{
XI WANG* , GUANGHUI LI* , YIMING LIU, WEIWEI YU and QIANG SUN \\ Department of Stomatology, The First Affiliated Hospital of Zhengzhou University, Zhengzhou, Henan 450052, P.R. China
}

Received January 28, 2017; Accepted March 7, 2017

DOI: 10.3892/br.2017.881

\begin{abstract}
To investigate the biocompatibility of the biomaterial, polylactic acid (PLA) with stem cells from human exfoliated deciduous teeth (SHED) and its induction of mineralization as a type of scaffold material. To determine the impacts of biomaterial PLA on proliferation and mineralization of SHED, the expression of surface molecules of SHED isolated and cultured in vitro was detected by flow cytometry. In addition, cell proliferation was measured using MTT and Edu assays, and the evaluation of mineralized differentiation was performed using Alizarin Red S staining. In addition, the expression levels of osteogenic marker genes were measured by reverse transcription-quantitative polymerase chain reaction (RT-qPCR) and western blot analysis. SHED were successfully isolated and identified. The MTT and Edu results indicated that the proliferation of SHED cultured in PLA and normal medium was not significantly different. The Alizarin Red S staining demonstrated that the mineralization capability was significantly higher in the SHED that were cultured in PLA medium. Furthermore, RT-qPCR and western blot analyses indicated that the expression levels of osteogenic marker genes were higher in the SHED cultured in PLA medium. These results suggested that PLA possesses good biocompatibility with SHED and may effectively induce the mineralization of SHED and serve as a scaffold material.
\end{abstract}

\section{Introduction}

The application of tissue engineering methods to construct biologically active and functional teeth has been the focus of oral medical research. Although the findings are currently

Correspondence to: Dr Guanghui Li or Dr Qiang Sun, Department of Stomatology, The First Affiliated Hospital of Zhengzhou University, 1 East Jian She Road, Zhengzhou, Henan 450052, P.R. China

E-mail: franklin1082@163.com

E-mail: sissy9288@163.com

${ }^{*}$ Contributed equally

Key words: polylactic acid, stem cells from human exfoliated deciduous teeth, osteogenic induction, biocompatibility insufficient to support their application in tooth regeneration, the intensive research on seed cells and scaffold materials has provided a prospective method for tooth tissue engineering. Duailibi et al (1) seeded tooth bud cells from 4-day-old rats on polyglycolic acid (PGA)-poly-L-lactate-co-glycolate (PLGA) scaffold material and implanted the tooth bud cells in the omenta of adult rat hosts. Dentin-, endodontium- and enamel-like structures were observed to have formed 12 weeks after implantation. Young et al (2) inoculated tooth bud cells from pigs after proliferation onto tooth-shaped bio-scaffold material PGA-PLGA and implanted these in the omenta of athymic rats, and found that tissue-engineered tooth crowns containing dentin and endodontium were formed.

A selection of scaffold materials, in addition to seed cells, are important in the construction of tissue-engineered teeth. Polylactic acid (PLA) is a typical biodegradable synthetic polymer (3). As it has reliable biosafety and is biodegradable and environmentally friendly, PLA has been widely applied as a medical polymer material (4-6) and is a commonly used biological scaffold material in tissue engineering $(7,8)$. In the current study, extracts of PLA were combined with stem cells from human exfoliated deciduous teeth (SHED) to evaluate the biocompatibility, with the aim of providing an experimental basis for subsequent tooth tissue engineering research.

\section{Materials and methods}

SHED culture. The present study was reviewed and approved by the Institutional Review Board and the Ethics Committee at Zhengzhou University (Zhengzhou, China) and was conducted following written consent being obtained from the guardians of all participants. Deciduous teeth that needed to be removed due to retention were collected and surface sterilized. Their crowns were carefully split in a sterile bench and their endodontium was removed, rinsed with sterile $0.01 \mathrm{~mol} / 1$ phosphate-buffered saline (PBS), sliced into small pieces of $\sim 1.0 \times 1.0 \times 1.0 \mathrm{~mm}$ and digested with $0.3 \%$ type I collagenase (Sigma-Aldrich; Merck KGaA, Darmstadt, Germany) at $37^{\circ} \mathrm{C}$ for $40 \mathrm{~min}$. The cell clumps were then prepared into a single cell suspension, passed through a sieve (pore size, $70 \mu \mathrm{m}$ ) and centrifuged at $120 \mathrm{x} g$ for $5 \mathrm{~min}$ at room temperature. The cells were washed again with sterile $0.01 \mathrm{~mol} / 1 \mathrm{PBS}$, resuspended into Gibco $\alpha$-minimum essential medium ( $\alpha$-MEM; Thermo Fisher Scientific, Inc., Waltham, MA, USA) containing Gibco $20 \%$ fetal bovine serum (FBS; Thermo Fisher Scientific, Inc.) 
and cultured in 5-ml flasks in an incubator supplemented with $5 \% \mathrm{CO}_{2}$ at $37^{\circ} \mathrm{C}$. The culture medium was refreshed every 3 days. Upon reaching $80 \%$ confluence, the cells were digested with $2.5 \mathrm{~g} / 1$ trypsin (Sigma-Aldrich; Merck KGaA) and passaged.

SHED phenotype identification. The P3 SHED at logarithmic phase were prepared as a single cell suspension. Subsequent to three washes with PBS, cells were resuspended in $1 \mathrm{ml}$ PBS containing 3\% FBS, counted and aliquoted into $1.5-\mathrm{ml}$ tubes with $100 \mu \mathrm{l}$ cell suspension in each tube to ensure that each tube had no less than $10^{5}$ cells. These cells were then incubated with $5 \mu$ l pre-prepared, light-protected, fluorescein isothiocyanateand phycoerythrin-labeled antibodies against human CD29 (1:500, cat. no. ab52971), CD105 (1:500, cat. no. ab44967), CD146 (1:200, cat. no. ab24577) and STRO-1 (1:100, cat. no. ab214086), all obtained from Abcam (Cambridge, MA, USA). The cells were then incubated for $1 \mathrm{~h}$ at room temperature in the dark, washed with PBS three times, resuspended and analyzed using flow cytometry (Beckman Coulter, Inc., Fullerton, CA, USA) according to manufacturer's instructions. Samples incubated with PBS served as the negative controls.

Preparation of PLA. The PLA ( $2 \mathrm{x} 1 \mathrm{~mm}$ size) was sterilized with ethylene oxide, mixed with $\alpha$-MEM containing 5\% FBS in a sterile tube to $100 \mathrm{~g} / 1$, incubated at $37^{\circ} \mathrm{C}$ for $72 \mathrm{~h}$ and stored at $4^{\circ} \mathrm{C}$ for future use.

Effect of PLA extract on SHED morphology. The P2 SHED were seeded onto 6-well plates and randomly divided into treatment and control groups with three repeats per group. Cells in the treatment and control groups were incubated with $100 \%$ PLA extract or with $\alpha$-MEM containing 5\% FBS, respectively, at $37^{\circ} \mathrm{C}$ in a humidified incubator supplemented with $5 \% \mathrm{CO}_{2}$ for 3 days. The cells were observed under an inverted phase contrast microscope (Olympus Corporation, Tokyo, Japan) for changes in cell adhesion and morphology.

Influence of PLA extract on the proliferation activity of SHED. The P3 SHED at logarithmic phase were resuspended in a medium of $100 \%$ PLA extract, a medium of 50\% PLA extract plus 50\% $\alpha$-MEM supplemented with 5\% FBS, and a medium of $100 \% \alpha$-MEM supplemented with 5\% FBS, seeded into 96-well plates with $100 \mu 1$ per well with eight duplicates for each and cultured at $37^{\circ} \mathrm{C}$ in a humidified incubator supplemented with $5 \% \mathrm{CO}_{2}$. At 1, 3, 5 and 7 days after culture, $20 \mu \mathrm{l}$ tetrazolium dye (MTT; Sigma-Aldrich; Merck KGaA) was added to each well and the cells were further cultured at $37^{\circ} \mathrm{C}$ for $4 \mathrm{~h}$. Subsequently, $50 \mu \mathrm{l}$ dimethyl sulfoxide (Sigma-Aldrich; Merck KGaA) was added to each well. The plate was oscillated at $600 \mathrm{rpm}$ for $10 \mathrm{~min}$ and the optical density value of each well was measured on a microplate reader (Bio-Rad Laboratories, Inc., Hercules, CA, USA) at a wavelength of $570 \mathrm{~nm}$.

The P3 SHED at logarithmic growth phase were prepared as a single cell suspension, seeded into each well of a 24-well plate with $4 \times 10^{3}$ cells per well and cultured to normal growth stage $(\sim 24 \mathrm{~h})$ at $37^{\circ} \mathrm{C}$ in a humidified incubator supplemented with $5 \% \mathrm{CO}_{2}$. After starving in serum-free $\alpha$-MEM for $12 \mathrm{~h}$, the cells were cultured in $\alpha$-MEM containing $10 \%$ FBS overnight, stained according to the EdU staining kit (Guangzhou
RiboBio Co., Ltd., Guangzhou, China) and photographed with a digital camera mounted on the microscope.

Effect of PLA extract on mineralization ability of SHED. The P3 SHED at logarithmic growth phase were seeded in 12 -well plates with $2 \times 10^{4}$ cells per well and randomly divided into treatment and control groups. Cells in the treatment and control groups were incubated with $100 \%$ PLA extract or with $\alpha$-MEM containing 5\% FBS, respectively, and cultured to $>80 \%$ confluency at $37^{\circ} \mathrm{C}$ in a humidified incubator supplemented with $5 \% \mathrm{CO}_{2}$. The medium was replaced with osteogenic medium, which was $\alpha$-MEM supplemented with 10\% FBS, $100 \mathrm{nM}$ dexamethasone (Sigma-Aldrich; Merck $\mathrm{KGaA}$ ), $50 \mu \mathrm{g} / \mathrm{ml}$ of ascorbic acid (Sigma-Aldrich; Merck $\mathrm{KGaA}$ ) and $5 \mathrm{mM} \beta$-glycerophosphate (Sigma-Aldrich; Merck $\mathrm{KGaA}$ ), half of the medium was replaced with osteogenic medium 3 days later and fully refreshed with osteogenic medium every 3 days subsequently. After 14 days of culture with the osteogenic supplements, the cells were fixed in 4\% paraformaldehyde, washed twice in PBS and incubated in $0.1 \%$ Alizarin Red S solution (Sigma-Aldrich; Merck KGaA) in Tris- $\mathrm{HCl}(\mathrm{pH} 8.3)$ at $37^{\circ} \mathrm{C}$ for $30 \mathrm{~min}$. After washing in PBS, the cells were observed and imaged under an inverted microscope. The nodule area per well was measured quantitatively using an image analysis system, Image-Pro plus 5.0 software (Media Cybernetics, Inc., Rockville, MD, USA).

$R T-q P C R$. The cultured SHED (14 days of osteogenic induction) were washed three times with PBS and sufficiently dried. The cells were cooled on ice and lysed with TRIzol reagent (Invitrogen; Thermo Fisher Scientific, Inc.). Total RNA was extracted using TRIzol reagent (Thermo Fisher Scientific, Inc.) according to the manufacturer's instructions. These RNA were immediately reverse transcribed into cDNA. RT-qPCR was performed in a final reaction volume of $20 \mu \mathrm{l}$ containing SYBR-Green PCR Master Mix (cat. no. 4309155; Thermo Fisher Scientific, Inc.), DNase-free water, primers and cDNA. The target genes runt-related transcription factor 2 (Runx2) and osterix (Osx) and the internal control, $\beta$-actin under the following conditions: Denaturation at $95^{\circ} \mathrm{C}$ for $3 \mathrm{~min}$; amplification ( 40 cycles) at $95^{\circ} \mathrm{C}$ for $15 \mathrm{sec}$ and $60^{\circ} \mathrm{C}$ for $30 \mathrm{sec}$. The PCR primer sequences are presented in Table I. Expression of each gene was monitored using an Applied Biosystems ${ }^{\circledR}$ StepOne ${ }^{\text {TM }}$ Real-Time PCR system (Thermo Fisher Scientific, Inc.). Differences in gene expression levels were calculated using the $2^{-\Delta \Delta \mathrm{Cq}}$ method (9) for relative quantification, and expressed as the fold change relative to the untreated control.

Western blot analysis. Whole cell lysates for western blotting were also extracted with lysis buffer and the total protein concentration was determined with Bradford protein assay kit (P0006; Beyotime Institute of Biotechnology, Shanghai, China) following the manufacturer's instructions. The samples of $50 \mu \mathrm{g}$ protein were loaded onto SDS gels and transferred to polyvinylidene fluoride membranes (EMD Millipore, Billerica, MA, USA). The membranes were blocked in 5\% non-fat dried milk in PBST (PBS+0.1\% Tween-20). The membranes were probed overnight with the following primary antibodies: anti-RUNX2 (1:100, cat. no. ab54868), anti-Osx (1:400, cat. no. ab57335) and anti- $\beta$-actin (1:800, cat. 
Table I. Polymerase chain reaction primer sequences.

\begin{tabular}{lll}
\hline Gene & Direction & \multicolumn{1}{c}{ Primers } \\
\hline Runx2 & Forward & CACTGGCGCTGCAACAAGA \\
& Reverse & CATTCCGGAGCTCAGCAGAATAA \\
Osx & Forward & TGGCGTCCTCCCTGCTTG \\
& Reverse & TGCTTTGCCCAGAGTTGTTG \\
\multirow{3}{*}{-actin } & Forward & TGGCACCCAGCACAATGAA \\
& Reverse & CTAAGTCATAGTCCGCCTAGAAGCA
\end{tabular}

Runx2, runt-related transcription factor 2; Osx, osterix.

no. ab3280). All antibodies were obtained from Abcam. The membranes were incubated at $37^{\circ} \mathrm{C}$ for $30 \mathrm{~min}$ with a horseradish peroxidase-conjugated secondary antibody $(1: 2,000$; cat. no. BA1056; Boster Biological Technology, Ltd., Wuhan, China). The membranes were developed using enhanced chemiluminescence (ECL) or ECL plus (GE Healthcare Life Sciences, Chalfont, UK) according to the manufacturer's instructions.

Statistical analysis. Each experiment was repeated three times and data were expressed as means \pm standard deviations. Data were analyzed using SPSS 17.0 statistical software (SPSS, Inc., Chicago, IL, USA). Differences between multiple groups were analyzed using Student's t-test following homogeneity test of variance. $\mathrm{P}<0.05$ was considered to indicate a statistically significant difference.

\section{Results}

SHED culture and identification. Slow attachment was observed during the primary SHED culture using an enzymatic digestion method; at $4 \mathrm{~h}$ of culture, only $60 \%$ of cells were attached. At $12 \mathrm{~h}$, cells had begun to stretch and at $48 \mathrm{~h}$, cells were substantially expanded. The passage cells grew well, and were passaged every 3-4 days. Cells at the center of a well exhibited unclear boundaries and were round or irregular, forming a nodular shape, while the majority of cells at the peripheral portion were small, spindle-shaped and fibroblast-like, and had 1-2 protrusions (Fig. 1A and B). Flow cytometry indicated that these cells were positive to mesenchymal stem cell surface markers, STRO-1, CD146, CD29 and CD105 (Fig. 1C-F).

Effect of PLA extract on SHED morphology. The morphology of cells cultured in PLA extract for 7 days was not significantly different when compared with that of cells cultured in $\alpha$-MEM supplemented with 5\% FBS, only certain cells were short and spindle-shaped, and had a relatively smaller volume (Fig. 1B).

Influence of PLA extract on the proliferation activity of SHED. EdU staining demonstrated that the nuclei of non-proliferating SHED were stained blue by Hoechst, while the nuclei of proliferating SHED were stained red due to incorporation of EdU. Thus, cells with red nuclei were positive, indicating that they were proliferating cells. The experimental results demonstrated that the number of proliferating cells was not significantly different among the three groups (Fig. 2A-D; $\mathrm{P}>0.1$ ), indicating that PLA extract did not significantly affect the proliferation activity of SHED. Furthermore, comparison of cell growth curves revealed that the proliferation of SHED cultured in PLA extracts was not significantly different from that of SHED cultured in $\alpha$-MEM supplemented with 5\% FBS (Fig. 2E).

Effect of PLA extract on mineralization ability of SHED. Alizarin Red S staining of cells after 14 days of culture under osteogenic culture conditions resulted in red mineralized nodules in irregular lumps or elongated shapes as observed under an optical microscope. In SHED cultured in $\alpha$-MEM supplemented with 5\% FBS, there were fewer mineralized nodules, which were scattered and darker in color. By contrast, there were significantly more mineralized nodules following culture in the medium with PLA extract, the nodules were larger, more concentrated, and lighter in color (Fig. 3A and B). Quantitative analysis of the Alizarin Red $S$ staining results indicated that the number of mineralized nodules of SHED cultured in PLA extract was significantly higher than that of SHED cultured in $\alpha$-MEM supplemented with 5\% FBS (Fig. 3C; $\mathrm{P}<0.05$ ). The expression levels of osteogenesis-associated genes at 14 days of osteogenic induction were detected using RT-qPCR. The results demonstrated that following osteogenic induction, the expression levels of RUNX2 and Osx were increased in the treatment groups, although these increases were significantly higher in SHED cultured in medium containing PLA extract (Fig. 3D and E; $\mathrm{P}<0.05)$. Similarly, western blot analysis also indicated that the expression levels of these proteins were increased following osteogenic induction, although these increases were also significantly higher in cells cultured in medium containing PLA extract (Fig. 3F; P<0.05). These results indicated that the osteogenic differentiation ability was significantly greater in SHED cultured in medium containing PLA extract.

\section{Discussion}

The key issues in tissue engineering are seed cells, scaffold biomaterials and cytokines. For tissue engineering research, it is particularly important to select the appropriate seed cells and scaffold biomaterials (10). PLA is an excellent biocompatible and biodegradable polymer. In an aqueous environment, PLA is degraded by simply hydrolyzing the ester bond to form its final degradation product, lactic acid, which is converted into pyruvate in the tricarboxylic acid cycle and eventually excreted in the form of $\mathrm{CO}_{2}$ and $\mathrm{H}_{2} \mathrm{O}(11,12)$. Effective stem cell proliferation and culture on biodegradable material PLA scaffolds have been reported $(13,14)$. Using PLA as a tissue-engineering scaffold provides a porous structure inside the stent for cell growth and nutrient transport, as well as the mechanical strength and geometric shape to support and guide cell growth $(15,16)$.

Adult stem cells refer to specific stem cells present in adult tissues and organs. Under normal circumstances, these cells are in a resting state, and used to regenerate cells and maintain cellular homeostasis. When tissues are damaged, these stem cells proliferate and differentiate to the cell types 
A

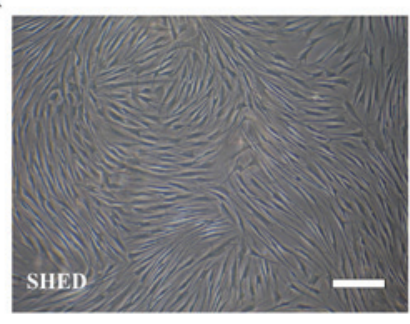

C

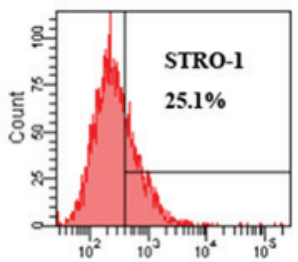

D

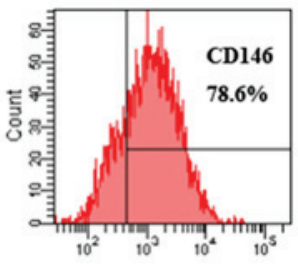

$\mathbf{F}$

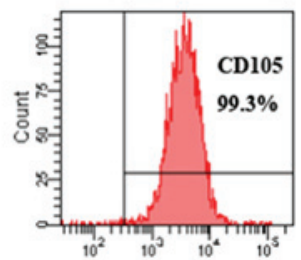

E

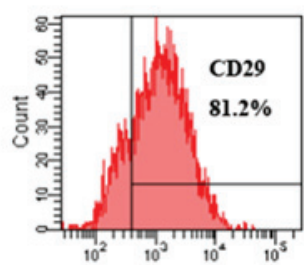

B

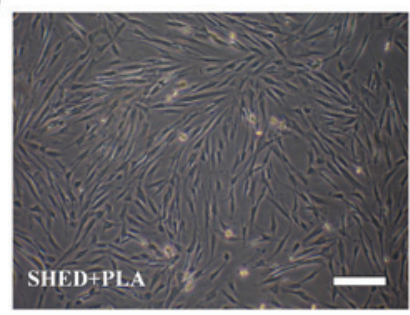

Figure 1. Cell morphology and FCM analysis of SHED. (A) Cell morphology of SHED incubated with $\alpha$-MEM containing 5\% FBS. (B) Cell morphology of SHED incubated with 100\% PLA extract. (C-F) Representative images of FCM data for SHED. Scale bars, $50 \mu \mathrm{m}$. FCM, flow cytometric; SHED, stem cells from human exfoliated deciduous teeth; PLA, polylactic acid; $\alpha$-MEM, $\alpha$-minimum essential medium; CD146, cluster of differentiation 146.

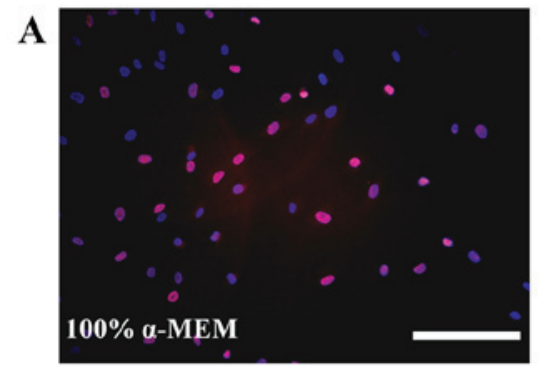

D

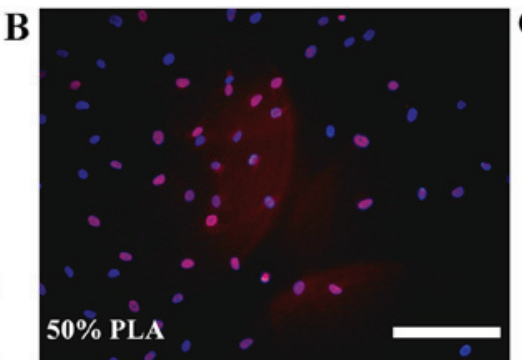

$\mathbf{E}$
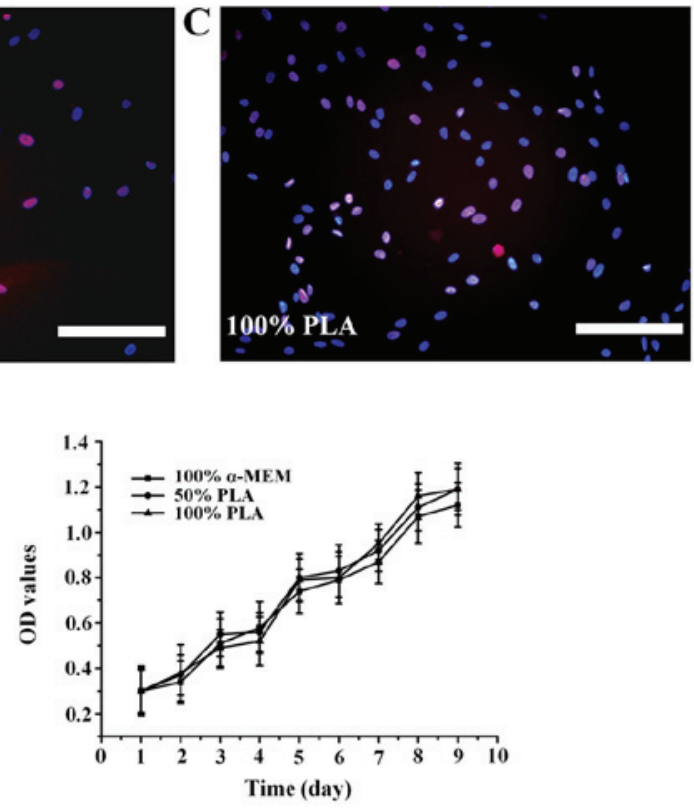

Figure 2. Proliferation ability of SHED cultured in normal medium and media with PLA extract. (A-C) Representative EdU staining of SHED cultured in normal medium and media with varying quantities of PLA extract. Cells with red nuclei are proliferating cells. (D) Percentage of EdU-positive cells (number of red nuclei). (E) Growth curves for SHED cultured in different media were analyzed using the MTT assay. The experimental results demonstrated that the number of proliferating cells was not significantly different between the three groups $(\mathrm{P}>0.1)$. The data shown represent mean \pm standard deviation. Scale bars, $50 \mu \mathrm{m}$. SHED, stem cells from human exfoliated deciduous teeth; PLA, polylactic acid; $\alpha$-MEM, $\alpha$-minimum essential medium; OD, optical density.

in the tissue, participating in tissue repair and reconstruction. In 2000, Gronthos et al (17) isolated cells with characteristics of stem cells from the endodontium. Later, Shi and Gronthos subcutaneously implanted a mix of single cell suspension 
A

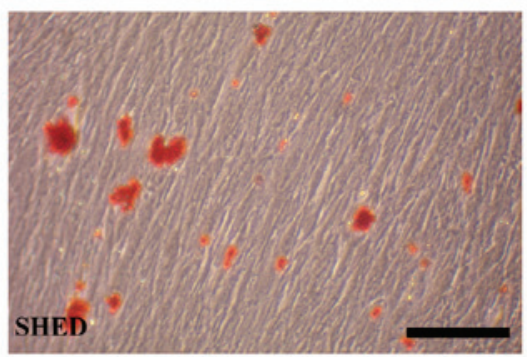

C

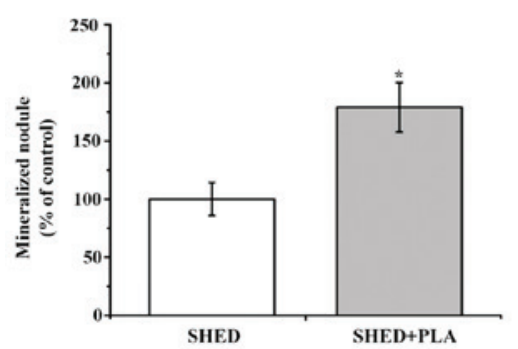

$\mathbf{E}$

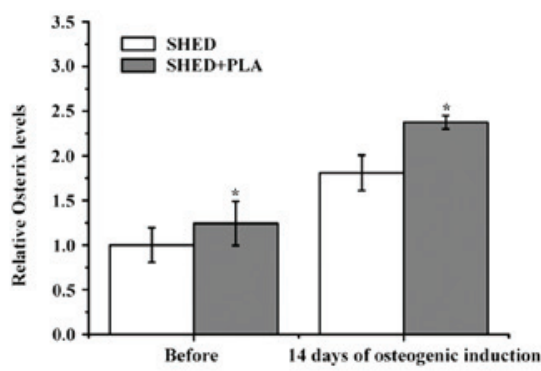

B

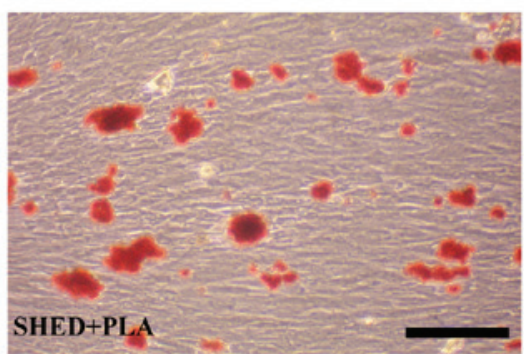

D

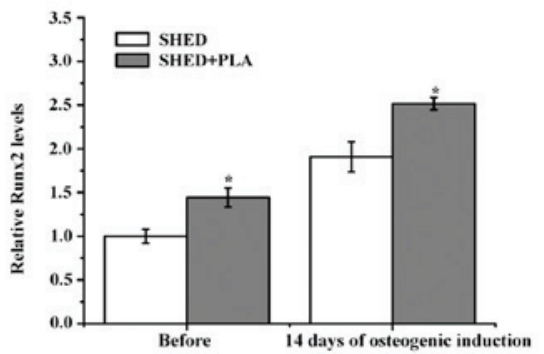

F
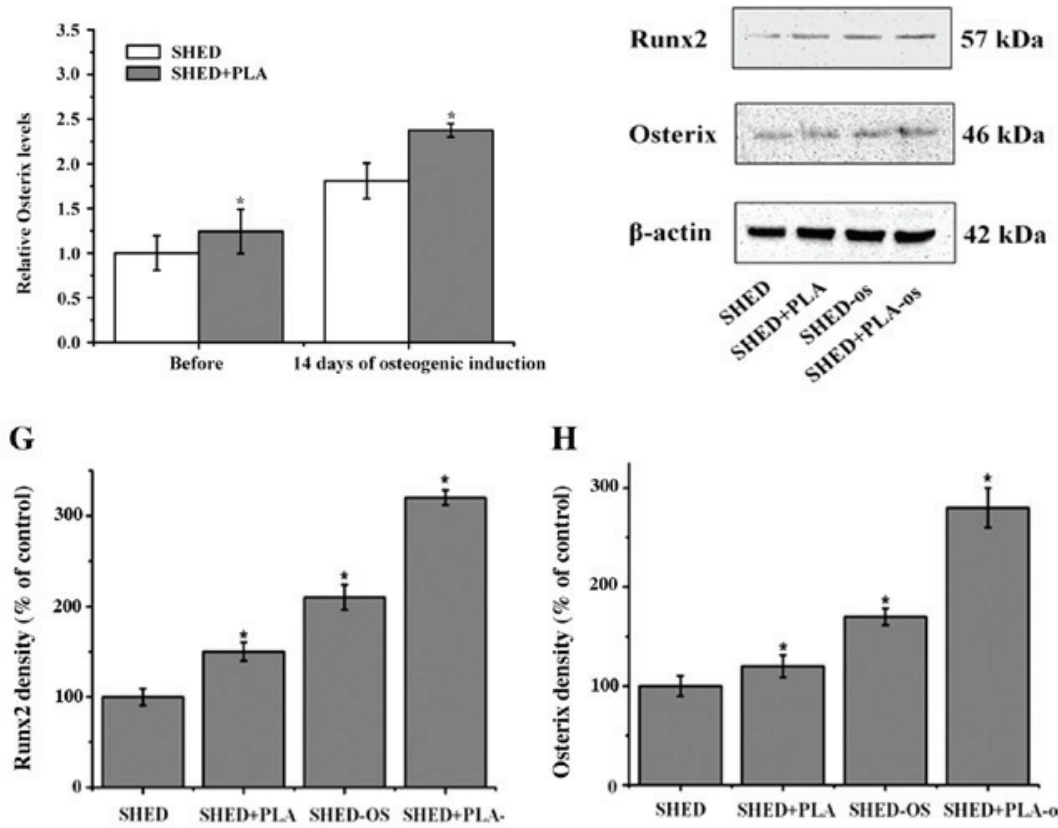

H

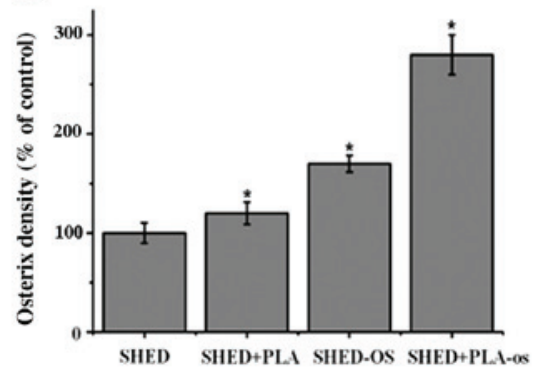

Figure 3. Osteogenic differentiation ability of SHED cultured in normal medium and medium with PLA extract. (A and B) Mineralization nodules formed in SHED cultured in normal medium and medium with PLA extract. Scale bars, $50 \mu \mathrm{m}$. (C) The graph presents the statistically significant difference in the number of mineralization nodules between the groups. (D and E) The osteogenesis-associated gene expression profiles of SHED cultured in various media were detected using reverse transcription-quantitative polymerase chain reaction. The results indicated that following osteogenic induction, the expression levels of RUNX2 and osterix were increased in all treatment groups; however, these increases were significantly higher in SHED cultured in media containing PLA extract. The data are presented as means \pm standard deviation. (F) The expression of RUNX2 and osterix of SHED cultured in various media were analyzed by western blotting. Western blot analysis indicated that the expression levels of these proteins were increased following osteogenic induction; however, these increases were significantly higher in cells cultured in medium containing PLA extract. (G and H) Quantification of these data. "P<0.05 vs. SHED. SHED, stem cells from human exfoliated deciduous teeth; PLA, polylactic acid; RUNX2, runt-related transcription factor 2; os, osteogenesis.

and hydroxyapatite/tricalcium phosphate (HA/TCP) powder into immunodeficient mice, forming the dentin-endodontium complex-like structures (18), and first proposed the concept of dental pulp stem cells. In 2003, Miura et al (19) found pluripotent SHED, which revealed a novel field for dental pulp stem cell research. Furthermore, studies have shown that SHED are superior to the permanent dental pulp stem cells in terms of proliferation and mineralization $(20,21)$. SHED provide an improved source of seed cells for tooth tissue engineering. In the current study, SHED were isolated using an in vitro enzymatic digestion method and flow cytometry identified that these cells are positive to STRO-1, CD146, CD29 and CD105, and possess the phenotypic characteristics of mesenchymal stem cells. 
Morphological observation results indicated that SHED cultured in vitro in PLA extraction properly attach, grow and reproduce, and demonstrated that biomaterial PLA has good biocompatibility, and is not toxic and inhibitory to cells, as well as being conducive to cell growth. In the present study, PLA extract at $100 \mathrm{~g} / 1$ displayed improved characteristics for cell proliferation. MTT and EdU analyses demonstrated that SHED were capable of normal growth and proliferation in PLA extract, further proving that PLA is not toxic to cells and has good biocompatibility. SHED secrete calcium matrix and aggregate further, forming mineralized nodules, which is the foundation for forming osteogenic/dentogenic dentin (22). Thus, the ability of forming mineralized nodules is an important indicator for evaluating the function of dental pulp stem cells. Alizarin Red $\mathrm{S}$ staining revealed that the quantity of mineralized nodules was significantly greater in cells cultured in PLA extract than in cells cultured in $\alpha$-MEM supplemented with 5\% FBS, indicating that PLA has improved mineralization induction ability for SHED. Furthermore, RT-qPCR and western blot analyses indicated that the expression levels of osteogenic marker genes were increased in SHED cultured in PLA medium. Adhered stem cells with strong mineralization ability significantly promote the formation of tissue-engineered teeth. As obtaining SHED is relatively convenient and noninvasive, their application is more simple and feasible compared with the other adult stem cells.

In conclusion, the application of the biomaterial, PLA in tooth tissue engineering is feasible due to its good histocompatibility and ability to improve the mineralization rate of SHED. However, its construction form requires improvement in order to increase cell attachment rate and cell proliferation. Since the simulation of complex biological environments has not yet been fully achieved, human tooth tissue engineering remains in its infancy. The present study may provide an experimental basis upon which human tooth regeneration using tissue engineering techniques may be achieved. As PLA is biodegradable and discharged to the environment in the form of $\mathrm{CO}_{2}$ and $\mathrm{H}_{2} \mathrm{O}$, the current study hypothesizes that the material may be applied in the restoration of deciduous teeth following root canal treatment.

\section{Acknowledgements}

The present study was funded by the National Natural Science Foundation of China (grant nos. 31400839 and 81402231).

\section{References}

1. Duailibi MT, Duailibi SE, Young CS, Bartlett JD, Vacanti JP and Yelick PC: Bioengineered teeth from cultured rat tooth bud cells. J Dent Res 83: 523-528, 2004.

2. Young CS, Terada S, Vacanti JP, Honda M, Bartlett JD and Yelick PC: Tissue engineering of complex tooth structures on biodegradable polymer scaffolds. J Dent Res 81: 695-700, 2002.

3. Liu X, Holzwarth JM and Ma PX: Functionalized synthetic biodegradable polymer scaffolds for tissue engineering. Macromol Biosci 12: 911-919, 2012.
4. Gao W and Wang J: Synthetic micro/nanomotors in drug delivery. Nanoscale 6: 10486-10494, 2014.

5. Wiebe J, Nef HM and Hamm CW: Current status of bioresorbable scaffolds in the treatment of coronary artery disease. J Am Coll Cardiol 64: 2541-2551, 2014.

6. Vanaman M and Fabi SG: Décolletage: Regional Approaches with Injectable Fillers. Plast Reconstr Surg 136: 276S-281S, 2015.

7. Gentile P, Chiono V, Carmagnola I and Hatton PV: An overview of poly(lactic-co-glycolic) acid (PLGA)-based biomaterials for bone tissue engineering. Int J Mol Sci 15: 3640-3659, 2014.

8. Venkatesan J and Kim SK: Nano-hydroxyapatite composite biomaterials for bone tissue engineering - a review. J Biomed Nanotechnol 10: 3124-3140, 2014.

9. Livak KJ and Schmittgen TD: Analysis of relative gene expression data using real-time quantitative PCR and the 2(-Delta Delta C(T)) Method. Methods 25: 402-408, 2001.

10. Langer R and Vacanti JP: Tissue engineering. Science 260: 920-926, 1993

11. Jeong W, Shin EJ, Culkin DA, Hedrick JL and Waymouth RM: Zwitterionic polymerization: A kinetic strategy for the controlled synthesis of cyclic polylactide. J Am Chem Soc 131: 4884-4891, 2009.

12. Castro-Aguirre E, Iñiguez-Franco F, Samsudin H, Fang X and Auras R: Poly(lactic acid)-Mass production, processing, industrial applications, and end of life. Adv Drug Deliv Rev 107: 333-366, 2016.

13. Sudwilai T, Ng JJ, Boonkrai C, Israsena N, Chuangchote S and Supaphol P: Polypyrrole-coated electrospun poly(lactic acid) fibrous scaffold: Effects of coating on electrical conductivity and neural cell growth. J Biomater Sci Polym Ed 25: 1240-1252, 2014.

14. Vuornos K, Björninen M, Talvitie E, Paakinaho K, Kellomäki M, Huhtala H, Miettinen S, Seppänen-Kaijansinkko R and Haimi S: Human Adipose Stem Cells Differentiated on Braided Polylactide Scaffolds Is a Potential Approach for Tendon Tissue Engineering. Tissue Eng Part A 22: 513-523, 2016.

15. Kim IG, Hwang MP, Du P, Ko J, Ha CW, Do SH and Park K: Bioactive cell-derived matrices combined with polymer mesh scaffold for osteogenesis and bone healing. Biomaterials 50: 75-86, 2015.

16. Salerno A, Guarino V, Oliviero O, Ambrosio L and Domingo C: Bio-safe processing of polylactic-co-caprolactone and polylactic acid blends to fabricate fibrous porous scaffolds for in vitro mesenchymal stem cells adhesion and proliferation. Mater Sci Eng C 63: 512-521, 2016.

17. Gronthos S, Mankani M, Brahim J, Robey PG and Shi S: Postnatal human dental pulp stem cells (DPSCs) in vitro and in vivo. Proc Natl Acad Sci USA 97: 13625-13630, 2000.

18. Shi S and Gronthos S: Perivascular niche of postnatal mesenchymal stem cells in human bone marrow and dental pulp. J Bone Miner Res 18: 696-704, 2003.

19. Miura M, Gronthos S, Zhao M, Lu B, Fisher LW, Robey PG and Shi S: SHED: Stem cells from human exfoliated deciduous teeth. Proc Natl Acad Sci USA 100: 5807-5812, 2003.

20. Galler KM, Cavender A, Yuwono V, Dong H, Shi S, Schmalz G, Hartgerink JD and D'Souza RN: Self-assembling peptide amphiphile nanofibers as a scaffold for dental stem cells. Tissue Eng Part A 14: 2051-2058, 2008.

21. Cordeiro MM, Dong Z, Kaneko T, Zhang Z, Miyazawa M, Shi S, Smith AJ and Nör JE: Dental pulp tissue engineering with stem cells from exfoliated deciduous teeth. J Endod 34: 962-969, 2008.

22. Yamaza T, Miura Y, Bi Y, Liu Y, Akiyama K, Sonoyama W, Patel V, Gutkind S, Young M, Gronthos S, et al: Pharmacologic stem cell based intervention as a new approach to osteoporosis treatment in rodents. PLoS One 3: e2615, 2008. 